TRANSACTIONS OF THE

AMERICAN MATHEMATICAL SOCIETY

Volume 350, Number 4, April 1998, Pages 1663-1680

S 0002-9947(98)01916-3

\title{
COMULTIPLICATIONS ON FREE GROUPS AND WEDGES OF CIRCLES
}

\author{
MARTIN ARKOWITZ AND MAURICIO GUTIERREZ
}

\begin{abstract}
By means of the fundamental group functor, a co-H-space structure or a co-H-group structure on a wedge of circles is seen to be equivalent to a comultiplication or a cogroup structure on a free group $F$. We consider individual comultiplications on $F$ and their properties such as associativity, coloop structure, existence of inverses, etc. as well as the set of all comultiplications of $F$. For a comultiplication $m$ of $F$ we define a subset $\Delta_{m} \subseteq F$ of quasi-diagonal elements which is basic to our investigation of associativity. The subset $\Delta_{m}$ can be determined algorithmically and contains the set of diagonal elements $D_{m}$. We show that $D_{m}$ is a basis for the largest subgroup $A_{m}$ of $F$ on which $m$ is associative and that $A_{m}$ is a free factor of $F$. We also give necessary and sufficient conditions for a comultiplication $m$ on $F$ to be a coloop in terms of the Fox derivatives of $m$ with respect to a basis of $F$. In addition, we consider inverses of a comultiplication, the collection of cohomomorphisms between two free groups with comultiplication and the action of the group Aut $F$ on the set of comultiplications of $F$. We give many examples to illustrate these notions. We conclude by translating these results from comultiplications on free groups to co-H-space structures on wedges of circles.
\end{abstract}

\section{INTRODUCTION}

The notion of a group is basic to many branches of mathematics. This notion has been described in general categorical terms so that one can define a group-object, or more generally, an H-object, in a category. This can be formally dualized to obtain co-H-objects and co-H-group-objects. For the category whose objects are based topological spaces and whose morphisms are homotopy classes of maps, a co$\mathrm{H}$-object is just a co-H-space and a co-H-group-object is just a co-H-group, both of which are of fundamental importance in algebraic topology. In this paper we study co-H-objects and co-H-group-objects in the category of groups and homomorphisms.

We start in $\S 2$ with a discussion of categories with coproducts and the definition of co-H-objects in such categories. We distinguish various types of co-H-objects (associative, coloop, commutative, etc.) and prove some general facts about them. We then consider coproduct preserving functors between such categories and show

Received by the editors July 19, 1996.

1991 Mathematics Subject Classification. Primary 20E05, 55P45; Secondary 55P40, 18A30.

Key words and phrases. Comultiplication, co-H-space, co-H-group, cogroup, free group, equalizers, category with coproducts, wedge of circles.

Part of this work was done while the first-named author was a visitor at the University of Milan. In addition, the second-named author also visited Milan for a brief period. The authors would like to thank both the Department of Mathematics at the University of Milan in general, and Professor Renzo Piccinini in particular, for their hospitality.

(C)1998 American Mathematical Society 
that they carry co-H-objects into co-H-objects. In particular, a categorical equivalence induces an equivalence between the co-H-objects of the corresponding categories. This result is applied to the fundamental group functor from the category of Eilenberg-Mac Lane spaces $K(\pi, 1)$ to the category of groups. We then conclude that the co-H-space structures on wedges of circles are equivalent to co-H-objects in the category of groups.

In $\S 3$ we begin our study of co-H-objects and co-H-group-objects in the category of groups (called groups with comultiplication and cogroups, respectively). These were first considered by Kan [10] who proved that a group admits a comultiplication if and only if it is a free group. He also showed that the set of diagonal elements of a cogroup forms a basis of the free group. For any comultiplication $m$ of a free group $F$ of finite rank we define a finite set of generators $\Delta_{m}$ of $F$ called quasi-diagonal elements. This set is an invariant of the comultiplication and measures its complexity. It can be effectively computed for any concretely defined comultiplication $m$ and it plays a key role in our study of associativity.

In $\S 4$ we prove that $\Delta_{m}$ contains the set $D_{m}=\left\{d \mid m d=d^{\prime} d^{\prime \prime}\right\}$ of diagonal elements of $m$. Thus we have a method to find $D_{m}$ by direct testing of the finitely many elements of $\Delta_{m}$. We show that $D_{m}$ freely generates the largest subgroup $A_{m}$ of $F$ on which $m$ is associative and that, moreover, the group $A_{m}$ is a free factor of $F$. We next compute the sets $\Delta_{m}$ and $D_{m}$ for several examples of comultiplications. The sets of diagonal elements are then used to classify the cohomomorphisms from a cogroup to a group with comultiplication. In $\S 5$ we find necessary and sufficient conditions for a group with comultiplication $m$ to be a coloop, that is, for the induced binary operation on $\operatorname{hom}(F, G)$ to have the structure of an algebraic loop, for all groups $G$. We do this in terms of the Jacobian matrices of Fox derivatives of $m$ with respect to any given basis. From this, we determine when our examples are coloops. Next we study inverses and commutativity in $\S 6$. We present many examples to illustrate the possibilities for inverses: inverses may not exist, a right inverse may exist but not a left inverse, both may exist and not be equal, etc. We also discuss briefly a variation of commutativity called quasi-commutativity.

In the last section we translate the results of $\S \S 3-6$ on comultiplications on groups to comultiplications on wedges of circles $S^{1} \vee \cdots \vee S^{1}$ using the categorical equivalence of $\S 2$. We show among other things that any two associative comultiplications of $S^{1} \vee \cdots \vee S^{1}$ are equivalent and we determine the group of co-H-equivalences of a wedge of $r$ circles with an associative comultiplication. Finally we show that any non-simply-connected associative co-H-space admits infinitely many equivalence classes of comultiplications.

We would like to emphasize that, despite our use of categories, our approach is often computational and example-oriented. We give many concrete calculations and present numerous examples in this paper. This illustrates the different concepts and results. For the remainder of this section we fix our notation and terminology in group theory.

We use the following group-theoretic terminology: groups $G$ are written multiplicatively, 1 is the identity element and $\bar{g}$ is the inverse $g^{-1}, g \in G$. The commutator of $g$ and $h$ is $[g, h]=\bar{g} \bar{h} g h$. Given elements $g_{1}, \ldots, g_{2 p}$ in $G$, write

$$
\prod_{i=1}^{2 p} g_{i}{ }^{(i)}=g_{1} \bar{g}_{2} \cdots g_{2 p-1} \bar{g}_{2 p},
$$

where $(i)=(-1)^{i+1}$. 
If $G$ and $H$ are groups, let $G * H$ denote the free product of $G$ and $H$. An element $\xi \in G * H$ can be written as a product

$$
\xi=g_{1} h_{1} \cdots g_{p} h_{p},
$$

with $g_{i} \in G, h_{j} \in H$. We call $\xi$ reduced if $g_{2}, \ldots, g_{p} \neq 1$ and $h_{1}, \ldots, h_{p-1} \neq 1$. The elements $g_{1}$ and $h_{p}$ may or may not be trivial. If $G=H$ and $g \in G$, then $g^{\prime} \in G * G$ denotes the element $g$ regarded as in the first free factor of $G * G$ and $g^{\prime \prime} \in G * G$ if $g$ is regarded as in the second free factor.

If $a: G \rightarrow K$ and $b: H \rightarrow L$ are homomorphisms, then $a * b: G * H \rightarrow K * L$ is defined by

$$
(a * b)\left(g_{1} h_{1} \cdots g_{p} h_{p}\right)=a\left(g_{1}\right) b\left(h_{1}\right) \cdots a\left(g_{p}\right) b\left(h_{p}\right) ;
$$

in addition, if $K=L$ there is a homomorphism $a \cdot b: G * H \rightarrow K$ defined by

$$
(a \cdot b)\left(g_{1} h_{1} \cdots g_{p} h_{p}\right)=a\left(g_{1}\right) b\left(h_{1}\right) \cdots a\left(g_{p}\right) b\left(h_{p}\right) .
$$

If $a, b: G \rightarrow H$ are two group homomorphisms, the equalizer subgroup $E$ of $G$ is defined by

$$
E=\{g \mid g \in G, a(g)=b(g)\},
$$

and $E$ is the largest subgroup of $G$ on which $a$ and $b$ agree.

If $S \subseteq G$ is a subset, then $\langle S\rangle$ denotes the subgroup of $G$ generated by $S$ and $|S|$ denotes the cardinality of $S$. If $\langle S\rangle$ is free and $S$ is a basis of $\langle S\rangle$, we say that $S$ is an independent set.

\section{Co-H-Objects in a CAtegory}

The purpose of this section is to describe the main objects of study, namely co$\mathrm{H}$-objects, in general categorical terms. We do this not to achieve the most general formulation, but rather as a means of unifying our two basic classes of co-H-objects, co-H-structures on wedges of circles and comultiplications on groups.

Let $\mathcal{C}$ be a category with finite coproducts and let $\mathcal{M o r}(X, Y)$ denote the set of morphisms in $\mathcal{C}$ from object $X$ to object $Y$. All categories considered will have zero morphisms, that is $\mathcal{M}$ or $(X, Y)$ has a distinguished object denoted $e$ and $f e=$ $e g=e$ for all $f \in \mathcal{M}$ or $\left(Y, Y^{\prime}\right)$ and all $g \in \mathcal{M}$ or $\left(X^{\prime}, X\right)$. Denote the coproduct of $X$ and $Y$ by $X \sqcup Y$ and the injections by $i_{X}: X \rightarrow X \sqcup Y$ and $i_{Y}: Y \rightarrow X \sqcup Y$. The unique morphism $X \sqcup Y \rightarrow Z$ determined by $f: X \rightarrow Z$ and $g: Y \rightarrow Z$ is denoted by $f \cdot g$. If $f: X \rightarrow Z$ and $g: Y \rightarrow U$ then $f \sqcup g: X \sqcup Y \rightarrow Z \sqcup U$ is defined to be $\left(i_{Z} f\right) \cdot\left(i_{U} g\right)$. If $X$ is an object in $\mathcal{C}, e: X \rightarrow X$ denotes the zero morphism and $1: X \rightarrow X$ denotes the identity morphism. A morphism $m: X \rightarrow X \sqcup X$ such that $(1 \cdot e) m=1=(e \cdot 1) m: X \rightarrow X$ is called a comultiplication and the pair $(X, m)$ is called a co-H-object. If $i_{1}, i_{2}: X \rightarrow X \sqcup X$ are the two injections of the coproduct, the morphism $i_{2} \cdot i_{1}: X \sqcup X \rightarrow X \sqcup X$ that exchanges the two factors is denoted $\tau$. If $m: X \rightarrow X \sqcup X$ is a comultiplication then so is $\tau m: X \rightarrow X \sqcup X$. It is called the opposite comultiplication and it is denoted by $m^{o p}$.

Definition 2.1. If $X$ is a co-H-object with comultiplication $m: X \rightarrow X \sqcup X$, then

(1) $m$ is called associative if $(1 \sqcup m) m=(m \sqcup 1) m$.

(2) $l: X \rightarrow X$ is called a left inverse of $m$ if $(l \cdot 1) m=e$.

(3) $r: X \rightarrow X$ is called a right inverse of $m$ if $(1 \cdot r) m=e$.

(4) If $m$ is associative and has left inverse $l$ and right inverse $r$, then $(X, m, l, r)$ is called a co-H-group-object. 
(5) We say that $m$ is commutative if $m=m^{o p}$.

Notice that if $(X, m)$ is a co-H-object, then $m$ induces a binary operation on $\mathcal{M o r}(X, Y)$ defined by $f+g=(f \cdot g) m$, for $f, g \in \mathcal{M o r}(X, Y)$. The zero morphism $e: X \rightarrow Y$ is a two-sided unit for this binary operation. Furthermore if $h: Y \rightarrow Z$, the induced function $h_{*}: \mathcal{M o r}(X, Y) \rightarrow \mathcal{M o r}(X, Z)$ is a homomorphism of sets with binary operation and two-sided unit.

Proposition 2.2. (1) There exists a comultiplication $m$ on an object $X$ if and only if for all objects $Y$ the set $\mathcal{M o r}(X, Y)$ has a binary operation with two-sided unit such that, for any morphism $h: Y \rightarrow Z$, the $\operatorname{map}_{*}$ : $\mathcal{M o r}(X, Y) \rightarrow \mathcal{M o r}(X, Z)$ is a homomorphism.

(2) The comultiplication $m$ is associative if and only if the induced binary operation in $\operatorname{Mor}(X, Y)$ is associative for all $Y$.

(3) The comultiplication $m$ has a left (resp., a right) inverse if and only if the induced binary operation on $\mathcal{M}$ or $(X, Y)$ has a left (resp., a right) inverse, for all $Y$.

(4) The co-H-object $(X, m)$ is a co-H-group-object if and only if $\mathcal{M} \operatorname{or}(X, Y)$ with the induced binary operation is a group, for all $Y$.

(5) The co-H-object $(X, m)$ is commutative if and only if the induced operation on $\operatorname{Mor}(X, Y)$ is commutative, for all $Y$.

For an indication of the proof of (1) see [1, Prop. 1.6]. The proofs for the other parts are straightforward.

It follows that a left inverse of an associative comultiplication is also a right inverse. Another property of a binary operation that we consider is the following. Let $L$ be a set with an additively denoted binary operation with two-sided unit. We say that $L$ is a loop if, for all $a, b \in L$, the equations $a+x=b$ and $y+a=b$ have unique solutions $x$ and $y$ in $L$.

Now let $\mathcal{C}$ be a category with finite coproducts, let $(X, m)$ be a co-H-object in $\mathcal{C}$ and let $\nabla=1 \cdot 1: X \sqcup X \rightarrow X$ be the folding map. Then $m$ (or $(X, m))$ is called a co-H-loop if $\mu_{1}=(1 \sqcup \nabla)(m \sqcup 1)$ and $\mu_{2}=(\nabla \sqcup 1)(1 \sqcup m): X \sqcup X \rightarrow X \sqcup X$ are equivalences in $\mathcal{C}[9]$.

Proposition 2.3. If $(X, m)$ is a co-H-object and $\mathcal{M}$ or $(X, Y)$ has the induced binary operation, then $(X, m)$ is a co-H-loop if and only if $\mathcal{M} \operatorname{or}(X, Y)$ is a loop, for all $Y$.

The proof given in [9, Thm. 2.3] for the topological category $\mathcal{T}$ of pointed topological spaces of the homotopy type of a CW-complex and homotopy classes of pointed maps carries over to an arbitrary category $\mathcal{C}$. It follows that every co-H-loop has a left and a right inverse and that every associative co-H-loop is a co-H-group-object.

If $(X, m)$ and $(Y, n)$ are co-H-objects of $\mathcal{C}$ and $f: X \rightarrow Y$ is a morphism, then $f$ is a co-H-morphism if

$$
n f=(f \sqcup f) m: X \rightarrow Y \sqcup Y .
$$

We write $f:(X, m) \rightarrow(Y, n)$ and $f$ induces a homomorphism $f^{*}: \mathcal{M o r}(Y, Z) \rightarrow$ $\mathcal{M o r}(X, Z)$ of sets with binary operation and unit. If $f$ is a co-H-morphism and an equivalence in $\mathcal{C}$, then we call $f$ a $c o$-H-equivalence. If $m$ is a comultiplication and $f: X \rightarrow X$ is an equivalence, we next define a new comultiplication $f \star m$ on $X$ by

$$
f \star m=(f \sqcup f) m f^{-1} .
$$


This is an operation of the group of self-equivalences of $X$ on the set of comultiplications of $X$. Call two comultiplications $m$ and $n$ of $X$ equivalent if $n=f \star m$ for some equivalence $f: X \rightarrow X$. This is then an equivalence relation in the set of comultiplications of $X$. Furthermore $f:(X, m) \rightarrow(X, f \star m)$ is a co-H-equivalence for every self-equivalence $f: X \rightarrow X$.

If $\mathcal{C}$ is a category with finite coproducts we can form a new category $\mathcal{C}_{c H}$ whose objects are co-H-objects $(X, m)$ of $\mathcal{C}$ and whose morphisms are co-H-morphisms $f:(X, m) \rightarrow(Y, n)$. This is called the primitive category in [6]. There are obvious full subcategories of $\mathcal{C}_{c H}$ consisting of co-H-objects which are associative, co-Hobjects which are co-H-loops, co-H-group-objects, etc. If $F: \mathcal{C} \rightarrow \mathcal{D}$ is a covariant functor which preserves coproducts, then $F$ induces a functor $F_{c H}: \mathcal{C}_{c H} \rightarrow \mathcal{D}_{c H}$ and corresponding functors for the various subcategories defined above. The following proposition is then clear.

Proposition 2.4. If $F: \mathcal{C} \rightarrow \mathcal{D}$ is a coproduct preserving covariant functor which is an equivalence of categories, then the induced functor is an equivalence $F_{c H}$ : $\mathcal{C}_{c H} \rightarrow \mathcal{D}_{c H}$ which induces equivalences of all the subcategories of $\mathcal{C}_{c H}$ mentioned above, with the corresponding subcategories of $\mathcal{D}_{c H}$. In particular, a co-H-object $(X, m)$ of $\mathcal{C}$ and the corresponding co-H-object $F_{c H}(X, m)$ of $\mathcal{D}$ have the same properties as co-H-objects.

We now specialize to specific categories. If $\mathcal{T}$ is the topological category, then $\mathcal{T}$ has coproducts given by the wedge $\vee$ of pointed spaces. In this category a co$\mathrm{H}$-object is called a co-H-space and a co-H-morphism is called a co-H-map. We mention in passing that the main motivation for considering co-H-loops is the following result [1]: any 1 -connected co-H-space is a co-H-loop.

Any full subcategory of $\mathcal{T}$ which is closed under the coproduct gives a category for which the notion of co-H-object makes sense. In particular let $\mathcal{K}$ be the full subcategory of $\mathcal{T}$ whose objects are Eilenberg-Mac Lane spaces $K(G, 1)$. Then $\mathcal{K}$ is closed under the wedge operation. Next, let $\mathcal{G}$ be the category of groups and homomorphisms. Then the free product of groups is a coproduct in $\mathcal{G}$. In this category a comultiplication $m: G \rightarrow G * G$ is still called a comultiplication but co-H-loops (or -group-objects, -morphisms, -isomorphisms) are called coloops (cogroups, cohomomorphisms, coisomorphisms, respectively).

There is an equivalence of categories $F: \mathcal{K} \rightarrow \mathcal{G}$ given by the fundamental group functor, $F=\pi_{1}$, so that $F(K(G, 1))=G$ with inverse $F^{\prime}$ given by $F^{\prime}(G)=K(G, 1)$. Since $F$ preserves coproducts, $F$ induces an equivalence $F_{c H}: \mathcal{K}_{c H} \rightarrow \mathcal{G}_{c H}$ and, by Proposition 2.4, equivalences in the corresponding subcategories of $\mathcal{K}_{c H}$ and $\mathcal{G}_{c H}$. It is well-known [5], [10] that a group $G$ admits a comultiplication $m: G \rightarrow G * G$ if and only if $G$ is a free group (see Corollary 3.2). But if $G$ is a free group of rank $r$, then $K(G, 1)$ is a wedge of $r$ circles $S^{1} \vee \cdots \vee S^{1}$. Thus we obtain the following result:

Proposition 2.5. There is an equivalence between the category of co-H-spaces $\left(S^{1} \vee \cdots \vee S^{1}, m\right)$ and co-H-maps and the category of free groups of finite rank with comultiplications $(F, n)$ and cohomomorphisms which is given by the fundamental group functor. Under this equivalence associative comultiplications, co-H-loop comultiplications, inverses of a comultiplication, etc. on a wedge of circles correspond to the respective notions on the resulting free group. 


\section{Quasi-Diagonal Sets}

Suppose that $G$ is a group and that $\xi \in G * G$. It is convenient to write $\xi$ as an alternating product

$$
\xi=\prod_{i=1}^{p} g_{i}{ }^{\prime} \bar{h}_{i}^{\prime \prime}, \quad g_{i}, h_{i} \in G .
$$

Then the maps $1 \cdot e, e \cdot 1: G * G \rightarrow G$ are given by $(1 \cdot e) \xi=\prod g_{i}$ and $(e \cdot 1) \xi=\prod \bar{h}_{i}$. We introduce the following notation: $E_{G}$ is the equalizer of $1 \cdot e$ and $e \cdot 1$, and $\pi: E_{G} \rightarrow G$ is the restriction of either $1 \cdot e$ or $e \cdot 1$ to $E_{G}$. Thus,

$$
E_{G}=\left\{\xi \mid \xi=\prod_{i=1}^{p} g_{i}{ }^{\prime} \bar{h}_{i}^{\prime \prime}, h_{p} \cdots h_{1} g_{1} \cdots g_{p}=1\right\}
$$

and $\pi(\xi)=\prod g_{i}=\prod \bar{h}_{i}$. Let $\xi_{u}=u^{\prime} u^{\prime \prime} \in E_{G}$, where $u \in G$ and $\Xi_{G}=\left\{\xi_{u} \mid u \neq 1\right\}$.

The following result [5] is based on ideas attributed to M. Kneser.

Proposition 3.1. The group $E_{G}$ is free with basis $\Xi_{G}$.

Proof. It is clear that the set $\Xi_{G}$ is an independent set. In order to write any expression $\xi=\prod g_{i}{ }^{\prime} \bar{h}_{i}^{\prime \prime}$ which satisfies $h_{p} \cdots h_{1} g_{1} \cdots g_{p}=1$ as a product of the $\xi_{u}$ and their inverses, we use the following simple algorithm: for $1 \leq i \leq 2 p$, define $\delta_{i}$ by the formulas

$$
\begin{aligned}
\delta_{2 k} & =h_{k} \cdots h_{1} g_{1} \cdots g_{k}, \\
\delta_{2 k+1} & =\delta_{2 k} g_{k+1} .
\end{aligned}
$$

Thus $\delta_{1}=g_{1}, \delta_{2}=h_{1} g_{1}, \ldots$ and $\delta_{2 p}=1$. Now one checks that $\xi$ is the alternating product

$$
\xi=\prod_{i=1}^{2 p} \xi_{\delta_{i}}^{(i)} .
$$

Note that

$$
\pi(\xi)=g_{1} \cdots g_{p}=\prod_{i=1}^{2 p} \delta_{i}^{(i)} .
$$

Neither formula (1) nor (2) is necessarily in reduced form. As a consequence of Proposition 3.1 we obtain a result of Kan [10, Cor. 3.11] (see also [5, Thm. 1.4]).

Corollary 3.2. A comultiplication $m$ of a group $G$ is a right inverse of $\pi: E_{G} \rightarrow$ $G$. Consequently a group $G$ admits a comultiplication if and only if $G$ is free.

Proof. The comultiplication $m: G \rightarrow G * G$ satisfies $(1 \cdot e) m=(e \cdot 1) m=1$; the first equality implies that $m: G \rightarrow E_{G}$, the second, that $\pi m=1$. Clearly $m$ is monic, so that $G$ is isomorphic to the subgroup $m(G) \subseteq E_{G}$ and hence a free group. Conversely, if $G$ is free with basis $X$, then $m(x)=x^{\prime} x^{\prime \prime}, x \in X$, defines a comultiplication of $G$.

Consequently we consider only free groups $F$ in the rest of the paper. The above result characterizes comultiplications as sections (i.e., right-inverses) of the map $\pi: E_{F} \rightarrow F$. We will consider them in this way wherever convenient. When we 
wish to distinguish the comultiplication $m$ from the section, we shall write the latter as $\widetilde{m}: F \rightarrow E_{F}$.

Remarks 3.3. (1) The proof of the proposition provides us with an algorithm to write any comultiplication $m$ on $F$ as $m(x)=\prod \xi_{\delta_{i}(x)}^{(i)}$ for each element $x$ in a basis $X$ of $F$. This will be used in the examples below.

(2) As noted, the expression in formula (1) is not necessarily in reduced form. However if $\xi=\prod_{i=1}^{p} g_{i}{ }^{\prime} \bar{h}_{i}^{\prime \prime}$ is in reduced form, then, in (1), $\delta_{i} \neq \delta_{i+1}$ for all $i<2 p-1$. Thus to put (1) in reduced form it is only necessary to delete those $\xi_{\delta_{i}}^{(i)}$ for which $\delta_{i}=1$. By gathering like terms in (1) we obtain a reduced expression of the form $\prod_{k} \xi_{\gamma_{k}}^{\epsilon_{k}}$, where $\epsilon_{k} \neq 0$ and $\gamma_{k} \neq \gamma_{k+1}$. This will be used in the examples below.

(3) In [8] Ganea gives an alternative approach to comultiplication of spaces. We briefly summarize his results and show their connections to the ideas of Proposition 3.1 and Corollary 3.2. If $\Sigma$ denotes the reduced suspension functor, $\Omega$ the loop space functor and $W$ is a connected CW-complex, define $\nu: \Sigma \Omega W \rightarrow W$ by $\nu(t, \omega)=\omega(t)$. The sections $\mu: W \rightarrow \Sigma \Omega W$ of $\nu$ are called coretractions. The pinching map $\sigma: \Sigma \Omega W \rightarrow \Sigma \Omega W \vee \Sigma \Omega W$ defines a co-H-structure. If $\rho: \Sigma \Omega W \rightarrow W \vee W$ is the composite $(\nu \vee \nu) \sigma$, then the formula $m=\rho \mu$ gives a one-to-one correspondence between sections $\mu: W \rightarrow \Sigma \Omega W$ and comultiplications $m: W \rightarrow W \vee W$ (see [1, $\S 4]$ or $[8, \S 1])$.

Let $G$ denote the group $\pi_{1}(W)$ and let $E_{W}$ denote the group $\pi_{1}(\Sigma \Omega W)$. Each element $g \in G$ is represented by a loop $\gamma_{g} \in \Omega W$. Define $\psi_{g}:[0,1] \rightarrow \Sigma \Omega W$ by $\psi_{g}(t)=\left(t, \gamma_{g}\right)$. Then $\psi_{g}$ can be viewed as an element of $E_{W}$. It is elementary that $E_{W}$ is free with basis $\Psi_{W}=\left\{\psi_{g} \mid g \neq 1\right\}$ and that $\nu_{*}\left(\psi_{g}\right)=g, g \neq 1$. The map $\sigma_{*}: E_{W} \rightarrow E_{W} * E_{W}$ is $\sigma_{*}\left(\psi_{g}\right)=\psi_{g}{ }^{\prime} \psi_{g}{ }^{\prime \prime}$, so that

$$
\rho\left(\psi_{g}\right)=\left(\nu_{*} * \nu_{*}\right) \sigma_{*}\left(\psi_{g}\right)=\nu_{*}\left(\psi_{g}\right)^{\prime} \nu_{*}\left(\psi_{g}\right)^{\prime \prime}=g^{\prime} g^{\prime \prime}=\xi_{g} \in E_{G} .
$$

Thus by Proposition 3.1, $\rho_{*}$ is a basis preserving isomorphism $E_{W} \rightarrow E_{G}$ such that $\pi \rho_{*}=\nu_{*}$. This allows us to identify the triple $\left(E_{W}, \Psi_{W}, \nu\right)$ with the corresponding triple $\left(E_{G}, \Xi_{G}, \pi\right)$ and sections $\mu: G \rightarrow E_{W}$ of $\nu_{*}$ with comultiplications $m: G \rightarrow$ $G * G$.

We illustrate Remarks (1) and (2) with some examples and we will return to Remark (3) in $\S 4$.

Examples 3.4. (1) Let $\xi=x^{\prime} x^{\prime \prime}\left[x^{\prime}, x^{\prime \prime}\right]=x^{\prime} x^{\prime \prime} \bar{x}^{\prime} \bar{x}^{\prime \prime} x^{\prime} x^{\prime \prime}$, for some $x \in F$. Then $\xi=\xi_{x} \bar{\xi}_{1} \xi_{\bar{x}} \bar{\xi}_{1} \xi_{x} \bar{\xi}_{1}=\xi_{x} \xi_{\bar{x}} \xi_{x}$, and the latter expression is reduced.

(2) If $F=\mathbb{Z}$ is a free group of rank 1 with basis $X=\{x\}$, then to simplify notation we let $\xi_{n}=\xi_{x^{n}}$. Set $\xi={x^{\prime}}^{3} x^{\prime \prime} x^{\prime-2} \in E_{F}$. Then in reduced form $\xi=\xi_{3} \bar{\xi}_{2}$.

(3) If $F$ is free of rank 2 with basis $X=\{x, y\}$ and $\xi=y^{\prime} y^{\prime \prime}\left[y^{\prime}, x^{\prime \prime}\right] \in E_{F}$, then $\xi=\xi_{y} \xi_{\bar{y}} \bar{\xi}_{x \bar{y}} \xi_{x}$.

Suppose $F$ is a free group with finite basis $X$ and a comultiplication $m$; then for each $w \in F$ we can write $m(w)=\xi_{\delta_{1}(w)}^{\epsilon_{1}} \cdots \xi_{\delta_{p}(w)}^{\epsilon_{p}}$ as a reduced expression by equation (1) and Remark 3.3(2). Thus the $\delta_{i}(w) \neq 1$ and the value of $p$ depends on $w$. We will write $p=p(w)$ when the dependency must be explicit.

Definition 3.5. The quasi-diagonal set $\Delta_{m}$ of $m$ is defined by

$$
\Delta_{m}=\left\{\delta_{i}(w) \mid w \in F, i=1,2, \ldots, p(w)\right\} .
$$


The reason for the name quasi-diagonal will become clear in the next section. An element $\delta \in F$ is quasi-diagonal if and only if $\xi_{\delta}$ appears in the reduced expression (as a product of elements in $\Xi_{F}$ ) of $m(w)$, for some $w \in F$.

Proposition 3.6. If $X$ is a basis of $F$ and $m$ is a comultiplication on $F$, then

$$
\Delta_{m}=\left\{\delta_{i}(x) \mid x \in X, i=1,2, \ldots, p(x)\right\}
$$

and $\Delta_{m}$ is a finite set of generators of $F$.

Proof. Equation (2) shows that $\Delta_{m}$ is a set of generators. Since $m$ is a homomorphism, only the $\delta_{i}(x)$ occur in the expression for $m(w), w \in F$. In that case $\left|\Delta_{m}\right| \leq \sum_{x \in X} p(x)<\infty$, since $X$ is finite.

Examples 3.7. In the following examples $F$ is a free group with basis $X$.

(1) Let $F=\mathbb{Z}$ with $X=\{x\}$ and let $m(x)=x^{\prime 3} x^{\prime \prime} x^{\prime-2}$. Then by Example $3.4(2), \Delta_{m}=\left\{x^{3}, x^{2}\right\}$. Notice that $\Delta_{m}$ generates $F$ but does not contain a basis of $F$.

(2) If $F$ is free of rank 2 and basis $X=\{x, y\}$ and $m$ is given by

$$
\begin{aligned}
& m x=x^{\prime} x^{\prime \prime}, \\
& m y=y^{\prime} y^{\prime \prime}\left[y^{\prime}, x^{\prime \prime}\right],
\end{aligned}
$$

then $\Delta_{m}=\{x, y, \bar{y}, x \bar{y}\}$ by Example 3.4(3). This example is attributed to Barratt in $[9$, p. 3]. The variation $n: F \rightarrow F * F$ defined by

$$
\begin{aligned}
& n x=x^{\prime} x^{\prime \prime}, \\
& n y=y^{\prime} y^{\prime \prime}\left[x^{\prime \prime}, y^{\prime}\right],
\end{aligned}
$$

gives $\Delta_{m}=\Delta_{n}$. Thus distinct comultiplications can have the same quasidiagonal set.

(3) Let $F$ be as in (2) and let $m$ be defined by

$$
\begin{aligned}
& m(x)=x^{2} y^{\prime} x^{\prime \prime} \bar{y}^{\prime} \bar{x}^{\prime}, \\
& m(y)=\bar{x}^{\prime \prime} y^{\prime} x^{\prime \prime} y^{\prime \prime} .
\end{aligned}
$$

Then $\Delta_{m}=\left\{x^{2} y, x y, x\right\}$.

(4) Let $F$ be of rank 3 with basis $\{x, y, z\}$ and $m$ be the comultiplication given by $m(x)=x^{\prime} x^{\prime \prime}, m(y)=y^{\prime} y^{\prime \prime}$ and

$$
m(z)=z^{\prime} z^{\prime \prime}\left[\bar{x}^{\prime},\left[\bar{x}^{\prime}, \bar{y}^{\prime \prime}\right]\right]=\xi_{z} \xi_{x} \bar{\xi}_{\bar{y} x} \xi_{\bar{y} x^{2}} \bar{\xi}_{x^{2}} \xi_{x} \bar{\xi}_{\bar{y} x} \xi_{\bar{y}} \xi_{\bar{y} x} \bar{\xi}_{x}
$$

Then $\Delta_{m}=\left\{x, y, z, \bar{y} x, \bar{y} x^{2}, x^{2}, \bar{y}\right\}$.

Note that in the above examples $\left|\Delta_{m}\right| \geq|X|$. The cardinality of $\Delta_{m}$ can be thought of as a measure of the complexity of the comultiplication $m$. In $\S 4$ we will see that a comultiplication is associative if and only if $\left|\Delta_{m}\right|=|X|$.

We show now that certain generating subsets of $F$ can be realized as the quasidiagonal subset of some comultiplication on $F$.

Proposition 3.8. Suppose that $F$ is a free group and that $X$ is a basis of $F$. Choose a factorization

$$
x=\prod_{i=1}^{p(x)} \delta_{i}(x)^{(i)}
$$


where $p(x) \geq 1$ and where the $\delta_{i}(x)$ are otherwise arbitrary (and possibly equal to 1). Then the formula

$$
m(x)=\prod_{i=1}^{p(x)} \xi_{\delta_{i}(x)}^{(i)}
$$

defines a comultiplication $F \rightarrow F * F$ with quasi-diagonal set

$$
\Delta_{m}=\left\{\delta_{i}(x) \mid \delta_{i}(x) \neq 1, x \in X, 1 \leq i \leq p(x)\right\} .
$$

Conversely, any comultiplication $m$ on $F$ determines a factorization

$$
x=\prod_{i=1}^{p(x)} \delta_{i}(x)^{(i)}
$$

for each $x \in X$ and $\delta_{i}(x) \in \Delta_{m} \cup\{1\}$.

The proof is trivial.

Example 3.9. Let $F$ have basis $X=\{x, y\}$ and write $x=\left(x^{2} y\right)(x y)^{-1}$ and $y=$ $1 x^{-1}(x y)$. The corresponding comultiplication is that in Example 3.7(3) and $\Delta_{m}=$ $\left\{x, x y, x^{2} y\right\}$.

We conclude this section with a discussion of cohomomorphisms. Let $f: F \rightarrow G$ be a homomorphism of free groups. We denote the basis elements of $E_{G}$ by $\eta_{v}=$ $v^{\prime} v^{\prime \prime}, v \in G$ and $v \neq 1$. Then the map $f * f: F * F \rightarrow G * G$ induces a map $f * f: E_{F} \rightarrow E_{G}$ satisfying

$$
(f * f)\left(\xi_{u}\right)=\eta_{f(u)},
$$

so basis elements of $E_{F}$ are mapped to basis elements of $E_{G}$ or 1. By Corollary 3.2 we have

Lemma 3.10. If $(F, m)$ and $(G, n)$ are groups with comultiplication, then a homomorphism $f: F \rightarrow G$ is a cohomomorphism if and only if the map $f * f: E_{F} \rightarrow E_{G}$ restricts to a map $m(F) \rightarrow n(G)$.

Proposition 3.11. Let $(F, m)$ and $(G, n)$ be two comultiplications, and let $f: F \rightarrow$ $G$ be a cohomomorphism. Then, if $f$ is injective, $f\left(\Delta_{m}\right) \subseteq \Delta_{n}$ and if $f$ is surjective, $\Delta_{n} \subseteq f\left(\Delta_{m}\right)$. Thus, if $f$ is a coisomorphism, $f \mid \Delta_{m}: \Delta_{m} \rightarrow \Delta_{n}$ is a bijection of sets.

Proof. Let $\xi_{i}=\delta_{i}^{\prime} \delta_{i}^{\prime \prime}$; if $\xi=\prod_{i=1}^{p} \xi_{i}^{\epsilon_{i}}$ is a reduced expression and $f$ is injective, then $(f * f)(\xi)$ is also reduced. So if $\xi_{\delta}$ occurs in an element of $m(F)$, then $\eta_{f(\delta)}$ occurs in its image in $n(G)$. If $f$ is surjective, then $(f * f)(\xi)=\prod \eta_{f\left(\delta_{i}\right)}^{\epsilon_{i}}$ is not necessarily a reduced expression. Thus some of the $\eta_{f\left(\delta_{i}\right)}$ may not occur in the reduced expression; in that case the corresponding $f\left(\delta_{i}\right)$ will not appear in $\Delta_{n}$.

We remark that the constant map $e: F \rightarrow G$ shows that the inclusions of 3.11 do not hold for an arbitrary cohomorphism. Recall from $\S 2$ that two comultiplications $m$ and $n$ on the group $F$ are equivalent if $n=\theta \star m$ for some $\theta \in$ Aut $F$. In that case $\theta:(F, m) \rightarrow(F, n)$ is a coisomorphism.

Corollary 3.12. If $F$ is a nontrivial free group, then there exist infinitely many equivalence classes of comultiplications on $F$. 
Proof. If $F=\mathbb{Z}$ has rank 1 , then, since there are two automorphisms of $F$, there are infinitely many equivalence classes of comultiplications on $F$. Now suppose that $F$ has rank $>1$. By Proposition 3.11, equivalent comultiplications have quasidiagonal sets of the same size. Therefore, it suffices to exhibit infinitely many comultiplications $m_{k}$ on $F$ with $\left|\Delta_{m_{k}}\right|<\left|\Delta_{m_{k+1}}\right|$. Let $X$ be a basis of $F$ and $w$ be an element of $F$ which is not a power of a basis element. Choose $x_{0} \in X$ and define $m_{k}(x)=x^{\prime} x^{\prime \prime}, x \in X, x \neq x_{0}$, and

$$
m_{k}\left(x_{0}\right)=x_{0}{ }^{\prime} x_{0}{ }^{\prime \prime}\left[w^{\prime}, w^{\prime \prime}\right]\left[w^{2^{\prime}}, w^{2^{\prime \prime}}\right] \cdots\left[w^{k^{\prime}}, w^{k^{\prime \prime}}\right] .
$$

Then $\Delta_{m_{k}}=X \cup\left\{w, w^{-1}, w^{2}, w^{-2}, \ldots, w^{k}, w^{-k}\right\}$.

\section{Associativity}

Throughout this section $F$ is a free group of rank $r$ and $m: F \rightarrow F * F$ is a comultiplication. We start with an illustration of Remark 3.3(3), whose notation we use. We consider the pinching map $\sigma: E_{F} \rightarrow E_{F} * E_{F}, \sigma\left(\xi_{g}\right)=\xi_{g}{ }^{\prime} \xi_{g}{ }^{\prime \prime}$, for $\xi_{g} \in \Xi_{F}$. Thus $\sigma$ is a comultiplication on $E_{F}$. We denote by $\widetilde{m}: F \rightarrow E_{F}$ the section of $\pi$ determined by $m$.

Proposition 4.1. If $(F, m)$ is a free group with comultiplication $m$ and if $w$ is an element of $F$, then the following are equivalent:

(1) $(1 * m) m w=(m * 1) m w$ in $F * F * F$,

(2) $(\widetilde{m} * \widetilde{m}) m w=\sigma(\widetilde{m} w)$ in $E_{F} * E_{F}$,

(3) if $m w=\prod \xi_{\delta_{i}}^{\epsilon_{i}}$ is in reduced form, then $m \delta_{i}=\xi_{\delta_{i}}$, for all $i$.

Proof. (1) $\Rightarrow(2)$ : If $j: E_{F} \rightarrow F * F$ is the inclusion, the equalizer $E q$ of $j * \pi$ and $\pi * j: E_{F} * E_{F} \rightarrow F * F * F$ is the image $\sigma\left(E_{F}\right)$. The proof that $E q \subseteq \sigma\left(E_{F}\right)$ is essentially the same as the proof of Proposition 3.1 and the other inclusion is trivial. Since $(1 * m) m w=(\pi * j)(\widetilde{m} * \widetilde{m}) m w$ and $(m * 1) m w=(j * \pi)(\widetilde{m} * \widetilde{m}) m w$, the equality (1) implies that $(\widetilde{m} * \widetilde{m}) m w=\sigma \lambda$ for $\lambda \in E_{F}$. We apply $1 \cdot e: E_{F} * E_{F} \rightarrow E_{F}$ to both sides of this equality to get $\widetilde{m} w=\lambda$ and so we obtain (2).

(2) $\Rightarrow(3):$ If $m w=\prod \xi_{\delta_{i}}^{\epsilon_{i}}$ is reduced, then

$$
\prod\left(\widetilde{m}\left(\delta_{i}\right)^{\prime} \widetilde{m}\left(\delta_{i}\right)^{\prime \prime}\right)^{\epsilon_{i}}=\prod\left(\xi_{\delta_{i}}{ }^{\prime} \xi_{\delta_{i}}{ }^{\prime \prime}\right)^{\epsilon_{i}}
$$

and all expressions are in reduced form because both $\widetilde{m} * \widetilde{m}$ and $\sigma$ are monic. Thus $m\left(\delta_{i}\right)=\xi_{\delta_{i}}$ for all $i$ and we obtain (3).

$(3) \Rightarrow(1)$ : Both $(1 * m) m\left(\delta_{i}\right)$ and $(m * 1) m\left(\delta_{i}\right)$ equal $\delta_{i}{ }^{\prime} \delta_{i}{ }^{\prime \prime} \delta_{i}{ }^{\prime \prime \prime}$ and this extends to any product $w$ of the $\delta_{i}$.

Corollary 4.2. A comultiplication $m$ is associative if and only if $\widetilde{m}:(F, m) \rightarrow$ $\left(E_{F}, \sigma\right)$ is a cohomomorphism.

Proof. For $m$ is a cohomomorphism if and only if (2) holds for all $w$.

We note that Corollary 4.2 is an analog for groups of Theorem 2.2 of [8]. Proposition 4.1(3) motivates the following definitions.

Definition 4.3. Let $m$ be a comultiplication on a free group $F$.

(1) An element $d \in F$ is diagonal with respect to $m$ if $d \neq 1$ and $m(d)=d^{\prime} d^{\prime \prime}=\xi_{d}$.

(2) The diagonal set $D_{m} \subseteq F$ is the set of all diagonal elements.

(3) The associator subgroup $A_{m}$ of $m$ is the equalizer of $(1 * m) m$ and $(m * 1) m$ : $F \rightarrow F * F * F$. 
Clearly $m$ is associative if and only if $A_{m}=F$. In this case Kan [10] proved assertion (2) below, namely that $D_{m}$ is a basis for $F$.

Theorem 4.4. If $m: F \rightarrow F * F$ is a comultiplication on a free group of finite rank, then

(1) $D_{m} \subseteq \Delta_{m}$.

(2) $A_{m}$ is the free group with basis $D_{m}$.

(3) $A_{m}$ is a free factor of $F$, that is, $F \simeq A_{m} * Q$ for some subgroup $Q$ of $F$.

Proof. The elements $\delta$ of $\Delta_{m}$ are characterized by the fact that $\xi_{\delta}$ occurs in the reduced expression of $m(x) \in E_{F}$, where $x$ is a member of a basis of $F$. Thus, the subgroup $\Phi_{m}=\left\langle\left\{\xi_{\delta} \mid \delta \in \Delta_{m}\right\}\right\rangle \subseteq E_{F}$ is the smallest free factor of $E_{F}$ that contains $m(F)$. Clearly, if $m(d)=\xi_{d}$, then $\xi_{d} \in \Phi_{m}$ and so $d \in \Delta_{m}$. This proves our first statement. The second statement follows immediately from Proposition 4.1. Finally $\left\langle\left\{\xi_{d} \mid d \in D_{m}\right\}\right\rangle=m\left(A_{m}\right)$ by the previous result and it is also a free factor of $\Phi_{m}$. It follows by [11, p. 117, Ex. 31] that $m\left(A_{m}\right)$ is a free factor of $m(F)$, and consequently $A_{m}$ is a free factor of $F$.

Remarks 4.5. (1) By Theorem 4.4(1) and Proposition 3.1 (see also Remark 3.3(1)), we have an algorithm for finding the diagonal elements of a comultiplication. One first finds the quasi-diagonal set $\Delta_{m}$ as in the previous section and then determines, by direct checking, which elements of $\Delta_{m}$ are diagonal. This yields $D_{m}$ by the theorem.

(2) It follows from the theorem that $(F, m)$ is associative if and only if $\Delta_{m}$ is a basis for $F$ if and only if $\Delta_{m}=D_{m}$.

Corollary 4.6. The correspondence $m \mapsto D_{m}$ is a one-to-one correspondence between associative comultiplications on $F$ and bases of $F$.

Proof. A comultiplication $m$ is associative if and only if $D_{m}$ is a basis of $F$. Clearly, then, $D_{m}$ determines $m$.

Corollary 4.7. Suppose that $F$ is a free group.

(1) Any two associative comultiplications on $F$ are equivalent.

(2) If $F$ is free of rank 1 and $\{x\}$ is its basis, the only associative comultiplications are given by $m(x)=x^{\prime} x^{\prime \prime}$ and $m^{o p}(x)=x^{\prime \prime} x^{\prime}$.

Proof. By Corollary 4.6 the diagonal set $D_{m}$ characterizes $m$. Given two associative comultiplications $m$ and $n$, the sets $D_{m}$ and $D_{n}$ are two bases of $F$. Thus there is an automorphism $\theta$ of $F$ with $\theta\left(D_{m}\right)=D_{n}$. It follows that $n=\theta \star m$. The second assertion follows from (1).

We illustrate these results with the following

Examples 4.8. We refer to the Examples 3.7.

(1) Example 3.7(1) gives $\Delta_{m}=\left\{x^{2}, x^{3}\right\}$ and, by inspection, $D_{m}=\emptyset$.

(2) Example 3.7(2) gives $\Delta_{m}=\{x, y, \bar{y}, x \bar{y}\}$ and $D_{m}=\{x\}$.

(3) Example 3.7(3) gives $D_{m}=\emptyset$.

(4) Example 3.7(4) gives $D_{m}=\{x, y\}$.

(5) Here is a new example: $F$ is free with basis $\{x, y\}$ and comultiplication

$$
\begin{aligned}
& m x=x^{\prime} \bar{y}^{\prime} x^{\prime \prime} \bar{y}^{\prime \prime} y^{\prime} y^{\prime \prime} \bar{y}^{\prime} y^{\prime \prime} \bar{x}^{\prime \prime} y^{\prime} x^{\prime \prime} \bar{y}^{\prime \prime}, \\
& m y=y^{\prime} y^{\prime \prime} \bar{y}^{\prime} y^{\prime \prime} \bar{x}^{\prime \prime} y^{\prime} x^{\prime \prime} \bar{y}^{\prime \prime} .
\end{aligned}
$$


Then $\Delta_{m}=\left\{x \bar{y}, y, \bar{y}, x \bar{y}^{2}\right\}$ and, by inspection, $D_{m}=\{x \bar{y}\}$.

Remark 4.9. It follows from Theorem 4.4(2) that $m \mid A_{m}: A_{m} \rightarrow A_{m} * A_{m}$ is an associative comultiplication and that the inclusion $\left(A_{m}, m \mid A_{m}\right) \rightarrow(F, m)$ is a cohomomorphism. Moreover, $A_{m}$ is the largest subgroup of $F$ for which $m$ is associative. In Section 6 we prove that $m \mid A_{m}$ admits a two-sided inverse, so that $\left(A_{m}, m \mid A_{m}\right)$ is a cogroup. Also since $A_{m}$ is a free factor of $F$, there is a retraction homomorphism $r: F \rightarrow A_{m}$. We shall consider below when $r$ is a cohomomorphism.

Next we briefly revisit cohomomorphisms. Let $(F, m)$ and $(G, n)$ be two comultiplications, $f: F \rightarrow G$ a homomorphism and $D_{m} \subseteq F, D_{n} \subseteq G$ the diagonal sets. Then we have the following analog of Proposition 3.11:

Proposition 4.10. If $f:(F, m) \rightarrow(G, n)$ is a cohomomorphism, then $f\left(D_{m}\right) \subseteq$ $D_{n} \cup\{1\}$. If in addition $f$ is injective, $f\left(D_{m}\right) \subseteq D_{n}$. Consequently, if $f$ is a coisomorphism, $f: D_{m} \rightarrow D_{n}$ is a bijection.

Proof. If $d \in D_{m}$, then $\widetilde{m}(d)=\xi_{d}$ and $\widetilde{n}(f(d))=\xi_{f(d)}$. Thus either $f(d) \in D_{n}$ or $f(d)=1$.

Remarks 4.11. (1) In view of Proposition 3.11 it is natural to ask whether $D_{n}$ is contained in $f\left(D_{m}\right)$ if $f$ is an epimorphism. This is not true in general. For instance, let $(F, m)$ be the first example in $3.7(2)$, and $(G, n)$ be infinite cyclic with basis $D_{n}=\{y\}$. Then the map $f: F \rightarrow G$ defined by $f(x)=1$ and $f(y)=y$ is a cohomomorphism of $F$ onto $G$, but $f\left(D_{m}\right)=1$.

(2) It is untrue in general that $f\left(\Delta_{m}\right) \subseteq \Delta_{n} \cup\{1\}$. Take $(F, m)$ as in Example $3.7(4)$, where $F$ has a basis $\{x, y, z\}$. If $(G, n)$ is of rank 2 with basis $\{x, z\}=\Delta_{n}=$ $D_{n}$, then the map $f: F \rightarrow G$ defined by $f(x)=x, f(y)=1, f(z)=z$ defines a cohomomorphism with $f\left(\Delta_{m}\right)=\left\{x, 1, z, x^{2}\right\}$.

Corollary 4.12. If $m$ is associative, then there exists a one-to-one correspondence between the set $\operatorname{Cohom}((F, m),(G, n))$ of cohomomorphisms and the set of functions $D_{m} \rightarrow D_{n} \cup\{1\}$. If $r$ is the rank of $F$ and $s$ is the rank of $G$, then there exist exactly $(s+1)^{r}$ elements in $\operatorname{Cohom}((F, m),(G, n))$. The group $\operatorname{Coaut}(F, m)$ is isomorphic to $S_{r}$, the symmetric group in $r$ letters.

We next give a condition for a homomorphism to be a cohomomorphism and apply this to the retraction $r: F \rightarrow A_{m}$.

Proposition 4.13. Let $(F, m)$ and $(G, n)$ be two groups with comultiplication. Assume that a homomorphism $f: F \rightarrow G$ satisfies

$$
f\left(\Delta_{m}\right) \subseteq D_{n} \cup\{1\} .
$$

Then $f$ is a cohomomorphism. In particular, a retraction $r: F \rightarrow A_{m}$ satisfying $r\left(\Delta_{m}\right) \subseteq D_{m} \cup\{1\}$ is a cohomomorphism.

Proof. By Lemma 3.10, if $\xi \in m(F)$, then we must show that $(f * f) \xi \in n(G)$. Any such $\xi$ can be written as $\prod \xi_{\delta_{i}}^{\epsilon_{i}}, \delta_{i} \in \Delta_{m}$. By hypothesis $f\left(\delta_{i}\right)=d_{i} \in D_{n} \cup\{1\}$. Now

$$
(f * f) \xi=\prod \xi_{d_{i}}^{\epsilon_{i}} \in\left\langle\left\{\xi_{d} \mid d \in D_{n}\right\}\right\rangle=n\left(A_{n}\right) \subseteq n(G) .
$$

The assertion for retractions follows from this. 
In Example 3.7(2) the map $r(x)=x, r(y)=1$ defines a retraction satisfying 4.13. However, in Example 3.7(4) no such retraction can exist, since $r(y)=y \in D_{m}$ and $r(\bar{y})=\bar{y} \notin D_{m}$. In Example 4.8(5) the map $r(x \bar{y})=x \bar{y}, r(y)=1$ satisfies 4.13. Finally, the example in Remark 4.11(2) does not satisfy 4.13 even though $f$ is a cohomomorphism.

\section{Loop Structures}

In this section we consider the problem of deciding whether a comultiplication $m$ on a free group $F$ of finite rank is or is not a coloop. As noted in $\S 2$, this is equivalent to the question of when the induced binary operation on $\operatorname{hom}(F, G)$ yields a loop for all groups $G$, but the actual definition is in terms of the maps $\mu_{i}$. If $X$ is a basis of $F$ these maps are defined as follows:

$$
\left\{\begin{array}{l}
\mu_{1}\left(x^{\prime}\right)=m(x), \quad\left\{\begin{array}{l}
\mu_{2}\left(x^{\prime}\right)=x^{\prime}, \\
\mu_{1}\left(x^{\prime \prime}\right)=x^{\prime \prime},
\end{array} \mu_{2}\left(x^{\prime \prime}\right)=m(x),\right.
\end{array}\right.
$$

where $x \in X$. Then $m$ is a coloop if and only if $\mu_{1}$ and $\mu_{2}$ are automorphisms of $F * F$.

The following proposition is immediate.

Proposition 5.1. The comultiplication $m$ on the free group $F$ is a coloop if and only if for any basis $X$ of $F$ both sets $m(X) \cup X^{\prime \prime}$ and $X^{\prime} \cup m(X)$ are bases of $F * F$.

The group ring of $F$ is denoted by $\mathbb{Z}[F]$ and the augmentation homomorphism $a: \mathbb{Z}[F] \rightarrow \mathbb{Z}$ is defined by $a(w)=1$, for $w \in F$. The kernel of $a$ is called $I(F)$, the fundamental ideal, and it is a free $\mathbb{Z}[F]$-module with basis $\{x-1 \mid x \in X\}$, where $X$ is a free basis of $F$ (see [7]). If $w \in F$, then $w-1 \in I(F)$; consequently $w-1$ is a linear combination $\sum_{x \in X} \omega_{x}(x-1)$ with coefficients $\omega_{x}$ in $\mathbb{Z}[F]$. These coefficients are called the Fox derivatives $\partial w / \partial x$ of $w$. The equality $(u v-1)=(u-1)+u(v-1)$, for $u, v \in F$, gives a derivation formula

$$
\partial u v / \partial x=\partial u / \partial x+u \partial v / \partial x .
$$

The units of $\mathbb{Z}[F]$ are precisely $\pm w$ for all $w \in F$.

Lemma 5.2. If $X$ is a basis for the free group $F$, then:

(1) For $x \in X$ and $w \in F$, the derivative $\partial w / \partial x$ is a unit in $\mathbb{Z}[F]$ if and only if $w=u x^{ \pm 1} v, u, v \in\langle X-\{x\}\rangle$; that is, $x$ occurs exactly once in the reduced expression for $w$.

(2) If $Y \subseteq F$ is of cardinality $|X|$, then $Y$ is a basis if and only if the matrix $\|\partial y / \partial x\|$ is invertible.

Proof. The first statement follows from (2.8) of [7]; the second is the main result of [3].

In general, the matrix of Lemma 5.2(2) is called the Jacobian matrix of $Y$ with respect to $X$. Given $X$, we can use Lemma 5.2 to verify the conditions of Proposition 5.1. That is, we compute the Jacobian matrices (with respect to the basis $X^{\prime} \cup X^{\prime \prime}$ of $\left.F * F\right) J^{*}$ of $m(X) \cup X^{\prime \prime}$ and $J^{* *}$ of $X^{\prime} \cup m(X)$. Clearly these matrices are

$$
J^{*}=\left(\begin{array}{cc}
J^{\prime} & J^{\prime \prime} \\
O & I
\end{array}\right) \quad \text { and } \quad J^{* *}=\left(\begin{array}{cc}
I & O \\
J^{\prime} & J^{\prime \prime}
\end{array}\right)
$$


where $I$ is the identity matrix and the matrices $J^{\prime}$ and $J^{\prime \prime}$ are defined below. Proposition 5.1 and Lemma 5.2 state that $m$ is a coloop if and only if the matrices $J^{*}$ and $J^{* *}$ are invertible. Consequently, we have

Corollary 5.3. The free group $F$ with comultiplication $m$ is a coloop if and only if the Jacobian matrices

$$
J^{\prime}=\left\|\partial m(x) / \partial y^{\prime}\right\| \quad \text { and } \quad J^{\prime \prime}=\left\|\partial m(x) / \partial y^{\prime \prime}\right\|
$$

are invertible for all $x, y \in X$.

Examples 5.4. We use our earlier examples to illustrate the previous results.

(1) Example 3.4(2): $F=\mathbb{Z}$ is infinite cyclic with basis $X=\{x\}$, and $m(x)=$ $x^{\prime 3} x^{\prime \prime} x^{\prime-2}$. Then $X^{\prime} \cup m(X)$ is a basis of $F * F$, and $J^{\prime \prime}$ is invertible. However, $m(X) \cup X^{\prime \prime}$ is not a basis and $J^{\prime}$ is not invertible.

(2) Example 3.7(2): If $F$ is free with basis $X=\{x, y\}$ and $m(x)=x^{\prime} x^{\prime \prime}, m(y)=$ $y^{\prime} y^{\prime \prime}\left[y^{\prime}, x^{\prime \prime}\right]$, then neither $X^{\prime} \cup m(X)$ nor $m(X) \cup X^{\prime \prime}$ are bases of $F * F$.

These two examples are special cases of the following general result.

Proposition 5.5. If $F$ is a free group with basis $X, x_{0}$ is an element of $X$ and $m$ is a comultiplication such that $m(x)=x^{\prime} x^{\prime \prime}$ for all $x \neq x_{0}$, then $m$ is a coloop if and only if $m\left(x_{0}\right)$ has one occurrence of $x_{0}{ }^{\prime}$ and one occurrence of $x_{0}{ }^{\prime \prime}$, both with exponent +1 .

Proof. In this case the matrices $J^{\prime}$ and $J^{\prime \prime}$ are invertible if and only if $\partial m\left(x_{0}\right) / \partial x_{0}{ }^{\prime}$ and $\partial m\left(x_{0}\right) / \partial x_{0}{ }^{\prime \prime}$ are units.

Using the notation of Example 3.4(2), we have

Corollary 5.6. If $F$ is the infinite cyclic group with generator $x$, then the only coloops on $F$ are $m(x)=x^{\prime} x^{\prime \prime}=\xi_{1}$ and $m^{o p}(x)=x^{\prime \prime} x^{\prime}=\bar{\xi}_{-1}$.

\section{Inverses And Commutativity}

Suppose that $F$ is a free group of finite rank. If $l: F \rightarrow F$ is a homomorphism, then $l$ and the identity map 1 on $F$ determine a homomorphism $l \cdot 1: F * F \rightarrow F$. Its restriction to $E_{F}$ is also denoted by $l \cdot 1$. If $m: F \rightarrow F * F$ is a comultiplication, then $l$ is a left inverse for $m$ if and only if $(l \cdot 1) m=e$. If $X$ is a basis for $F$ and $m x=\prod g_{i}{ }^{\prime} h_{i}{ }^{\prime \prime}$ in reduced form, then $l: F \rightarrow F$ is a left inverse of $m$ if and only if $\prod l\left(g_{i}\right) h_{i}=1$ in $F$. Similar remarks apply to right inverses $r: F \rightarrow F$. If $m$ is associative, then $D_{m}$ is a basis of $F$ and $m d=d^{\prime} d^{\prime \prime}$ for each $d \in D_{m}$. It follows that $l$ must satisfy $l(d) d=1$, and so $l(d)=\bar{d}$. Conversely, this latter equation defines a left inverse $l$ of $m$. Similarly, $r(d)=\bar{d}$ defines a right inverse.

If $l: F \rightarrow F$ is a unique left inverse for a comultiplication $m: F \rightarrow F * F$, $r: F \rightarrow F$ is a unique right inverse for $m$ and $l=r$, then we say that $l$ is a unique two-sided inverse for $m$.

Summarizing, we have

Proposition 6.1. If $m$ is an associative comultiplication, then there is a unique two-sided inverse for $m$ defined by $l(d)=\bar{d}, d \in D_{m}$. In particular, any associative comultiplication of $F$ is a cogroup. 
The categorical remarks in $\S 2$ regarding inverses apply to free groups. In particular if $(F, m)$ is a coloop, there is a unique left inverse and a unique right inverse of $m$.

In the next result $[F, F]$ denotes the commutator subgroup of $F$.

Proposition 6.2. If $l: F \rightarrow F$ is a left inverse for a comultiplication $m: F \rightarrow$ $F * F$ and if $r$ is a right inverse, then

(1) $l(w) \equiv r(w) \equiv \bar{w}(\bmod [F, F])$, for all $w \in F$.

(2) $l(d)=r(d)=\bar{d}$, for all $d \in D_{m}$.

Proof. For (1), if $m w=\prod g_{i}{ }^{\prime} h_{i}{ }^{\prime \prime}$, then $w=\prod g_{i}=\prod h_{i}$ and, modulo $[F, F]$,

$$
1=\prod l\left(g_{i}\right) h_{i} \equiv \prod l\left(g_{i}\right) \prod h_{i}=l\left(\prod g_{i}\right) \prod h_{i}=l(w) w .
$$

Consequently $l(w) \equiv \bar{w}$ and similarly $r(w) \equiv \bar{w}$ modulo $[F, F]$. For the second assertion we proceed as in Proposition 6.1.

Corollary 6.3. If $F$ is infinite cyclic, then the homomorphism $l(a)=\bar{a}, a \in F$, is the unique two-sided inverse for every comultiplication on $F$.

Remark 6.4. Observe that for any $m,\left(A_{m}, m \mid A_{m}\right)$ is a cogroup.

Examples 6.5. (1) A comultiplication with unique two-sided inverse which is not a coloop: any comultiplication in $\mathbb{Z}$ except the two in Corollary 5.6.

(2) A comultiplication with no inverses: let $F$ have basis $\{x, y\}$ and

$$
\begin{aligned}
& m(x)=x^{\prime} x^{\prime \prime}, \\
& m(y)=y^{\prime} y^{\prime \prime} \bar{y}^{\prime} \bar{y}^{\prime \prime} \bar{x}^{\prime \prime} y^{\prime} x^{\prime \prime} y^{\prime \prime} .
\end{aligned}
$$

If $l$ and $r$ are the left and right inverses of $m$, then $l(x)=r(x)=\bar{x}$ by Proposition 6.2, and

$$
\begin{aligned}
l(y) y[l(y), x y] & =1, \\
{[\bar{y}, r(\bar{y})] x y \bar{x} r(y) } & =1 .
\end{aligned}
$$

We show that these two equations have no solution in $F$ by mapping $F$ to the symmetric group in three letters $S_{3}$ by the homomorphism $\theta: F \rightarrow S_{3}$ given by $\theta(x y)=(23)$ and $\theta(y)=(12)$. Then the equation for $\lambda=\theta(l(y))$ becomes

$$
(*) \quad \lambda(12) \lambda(23) \lambda(23)=1 .
$$

A simple ennumeration of possible cases shows that there is no solution $\lambda$ for (*). A similar argument using $\theta$ for the right inverse equation can be made.

(3) A comultiplication with right but not left inverse: we use the first comultiplication $m$ in Example 3.7(2). Any right inverse $r$ will have to satisfy $r(x)=\bar{x}$ by Proposition 6.2. For $r(y)$ we have $r(y)=\bar{y} x \bar{y} \bar{x} y$. It has been stated in [3, p. 9] that $m$ has no left inverse. Here is a short proof: if $l$ is a left inverse, then $l(y)$ must satisfy $l(y) y[l(y), x]=1$. Then $\eta: F \rightarrow S_{3}$ defined by $\eta(x)=(23)$ and $\eta(y)=(12)$ gives equation $(*)$ of the previous example. Thus $l$ does not exist. The comultiplication $m^{o p}$ has a left but not a right inverse.

(4) A comultiplication that admits right and left inverses which are distinct. Use the comultiplication of Example 3.7(3). Then the comultiplication has left and right inverses given by

$$
\left\{\begin{array} { l } 
{ l ( x ) = x \overline { y } \overline { x } y \overline { x } , } \\
{ l ( y ) = x \overline { y } \overline { x } , }
\end{array} \quad \left\{\begin{array}{l}
r(x)=\bar{y} \bar{x} y, \\
r(y)=\bar{y} x \bar{y} \bar{x} y .
\end{array}\right.\right.
$$


Notice that $m$ is not a coloop, since $m(X) \cup X^{\prime \prime}$ is not a basis of $F * F$.

(5) A comultiplication on a free group of rank 3 which is a coloop with distinct right and left inverses. Use the comultiplication of Example 3.7(4). This is a coloop because $m(z)=\xi_{z} \prod \xi_{u_{i}}^{\epsilon_{i}}, u_{i} \in\langle\{x, y\}\rangle$, and so $m$ has unique right and left inverses. However, they are not equal. For, by Proposition 6.2(2), $l(x)=r(x)=\bar{x}$ and $l(y)=r(y)=\bar{y}$, but

$$
l(z)=\bar{x} y x \bar{y} \bar{x} y \bar{x} \bar{y} x^{2} \bar{z} \text { and } r(z)=\bar{z} x \bar{y} \bar{x} y x \bar{y} x y \bar{x}^{2} .
$$

Remark 6.6. There are large families of comultiplications which have two-sided inverses. If $F$ has basis $\{x, y\}$, define $m$ by $m(x)=x^{\prime} x^{\prime \prime}$ and $m(y)=y^{\prime q+1} v y^{\prime \prime} \bar{v} k \bar{y}^{\prime} q$, where $v$ is a word in $x^{\prime}$ and $x^{\prime \prime}$ with arbitrary exponent sum $p$ in $x^{\prime}$ and zero exponent sum in $x^{\prime \prime}$. The factor $k$ is an arbitrary product of commutators $\left[x^{\prime a}, x^{\prime \prime b}\right]^{ \pm 1}$. A direct computation gives the two-sided inverse $l(x)=\bar{x}$ and $l(y)=x^{p} \bar{y} \bar{x}^{p}$. Notice that $l(y) \neq \bar{y}$.

In $\S 2$ we defined a morphism $\tau: X \sqcup X \rightarrow X \sqcup X$. In the category of groups we denote this map by $t: F * F \rightarrow F * F$, where $t\left(x^{\prime}\right)=x^{\prime \prime}$ and $t\left(x^{\prime \prime}\right)=x^{\prime}$. Then $t m=m^{o p}$ and $m$ is commutative if $m=m^{o p}$. The following proposition was proved in [5, Thm. 1.7].

Proposition 6.7 . If $m$ is a comultiplication on a nontrivial free group $F$, then $m$ is not commutative.

Proof. If $m w=\prod \xi_{\delta_{i}}^{\epsilon_{i}}$ is in reduced form, then $m^{o p} w=\prod \xi_{\bar{\delta}_{i}}^{-\epsilon_{i}} \neq m w$.

We consider a more general concept which is taken from the theory of $\mathrm{H}$-spaces [12, Prob. 34].

Definition 6.8. A comultiplication $m$ on $F$ is quasi-commutative if $t m=\theta \star m$ for some $\theta \in \operatorname{Aut} F$.

Clearly commutativity implies quasi-commutativity. Moreover, if $m$ is associative then $m$ is quasi-commutative. Indeed $l(d)=\bar{d}, d \in D_{m}$, defines an automorphism of $F$, and $t m=l \star m$.

We wish to characterize the quasi-commutative comultiplications on an infinite cyclic group $F$. Before doing that it is necessary to characterize all comultiplications on $F$. We use the notation of Example 3.4(2).

Lemma 6.9. If $F$ is infinite cyclic with basis $\{x\}$ and $\lambda=\prod_{j} \xi_{i_{j}}^{\epsilon_{j}}$ is a reduced expression in $E_{F}$, where $i_{j}, \epsilon_{j} \in \mathbb{Z}$, then $m(x)=\lambda$ defines a comultiplication if and only if $\sum_{j} i_{j} \epsilon_{j}=1$.

Proof. If $\lambda=\prod_{j} \xi_{i_{j}}^{\epsilon_{j}}$ is an arbitrary element in $E_{F}$, then $\pi(\lambda)=x^{s}$, where $s=$ $\sum_{j} i_{j} \epsilon_{j}$

Proposition 6.10. If $F$ is infinite cyclic with basis $\{x\}$ and $m$ is the comultiplication defined by $m(x)=\prod_{j=1}^{p} \xi_{i_{j}}^{\epsilon_{j}}$, then $m$ is quasi-commutative if and only if $i_{j}=i_{p+1-j}$ and $\epsilon_{j}=\epsilon_{p+1-j}$, for all $1 \leq j \leq p$.

Proof. The only non-trivial automorphism of $F$ is given by $l(x)=\bar{x}$. Let $m$ be a comultiplication defined by $m(x)=\prod_{j=1}^{p} \xi_{i_{j}}^{\epsilon_{j}}$, as in Lemma 6.9. Then $\operatorname{tm}(x)=$ $\prod \xi_{-i_{j}}^{-\epsilon_{j}}$ while $l \star m=\left(\prod \xi_{-i_{j}}^{\epsilon_{j}}\right)^{-1}$. The result follows. 


\section{CO-H-SPACES}

The purpose of this section is to translate earlier results on comultiplications of free groups into results on comultiplications on wedges of circles by using Proposition 2.5. The usual conventions of homotopy theory hold. In particular, by a map $V \rightarrow W$ is meant a homotopy class of maps $V \rightarrow W$ and $[V, W]$ denotes the set of all such homotopy classes.

We discuss $S^{1}$ first. By Lemma 6.9, there exist infinitely many comultiplications on the circle. Let $\sigma: S^{1} \rightarrow S^{1} \vee S^{1}$ be the standard suspension comultiplication obtained by regarding $S^{1}$ as the suspension of $S^{0}$.

The following is a translation of Corollaries 4.7, 5.6 and 6.3:

Proposition 7.1. (1) The comultiplications $\sigma$ and $\sigma^{o p}$ are the only associative comultiplications of $S^{1}$.

(2) The comultiplications $\sigma$ and $\sigma^{o p}$ are the only coloop comultiplications of $S^{1}$.

(3) The map $l: S^{1} \rightarrow S^{1}$ of degree -1 is a two-sided inverse for every comultiplication of $S^{1}$.

Remarks 7.2. (1) We note that (1) is an immediate consequence of (2) and (3), and that (3) can be proved directly since the induced binary operation in $\left[S^{1}, S^{1}\right]$ is a group operation.

(2) By Proposition 6.10, there exist comultiplications of $S^{1}$ which are not quasicommutative.

We now consider comultiplications on $S^{1} \vee \cdots \vee S^{1}$, a wedge of $r$ circles. Recall that two comultiplications $\phi$ and $\psi$ are equivalent if there is an equivalence $\theta$ : $S^{1} \vee \cdots \vee S^{1} \rightarrow S^{1} \vee \cdots \vee S^{1}$ with $\theta \star \phi=\psi$.

The following is an immediate consequence of Corollary 4.7, Corollary 4.12 and Proposition 6.1:

Theorem 7.3. (1) Any two associative comultiplications on a wedge of circles are equivalent.

(2) If $\phi$ is any associative comultiplication on a wedge of $r$ circles, then the group of co-H-equivalences of $\left(S^{1} \vee \cdots \vee S^{1}, \phi\right)$ is isomorphic to the symmetric group $S_{r}$.

(3) Every associative comultiplication $\phi$ on a wedge of circles has a two-sided inverse, and hence $\left(S^{1} \vee \cdots \vee S^{1}, \phi\right)$ is a co-H-group.

Remarks 7.4. There exist comultiplications on wedges of circles with the properties of the Examples 6.5(1)-(6).

From Corollary 3.12 we have

Lemma 7.5. There exist infinitely many equivalence classes of comultiplications on a wedge of circles.

The situation for a wedge of circles contrasts greatly with the simply connected case. For a wedge of circles there is only one equivalence class of associative comultiplications and there are infinitely many equivalence classes of comultiplications. On the other hand, there exist simply connected co-H-spaces $Y$ with infinitely many equivalence classes of associative comultiplications [2, Ex. 6.6], and clearly $S^{n}, n \geq 2$, admits a unique comultiplication.

Finally, Lemma 7.5 generalizes to certain non-simply connected spaces. 
Theorem 7.6. Let $W$ be a non-simply-connected associative co-H-space of finite type. Then there are infinitely many equivalence classes of comultiplications on $W$.

Proof. By the Berstein-Dror theorem [4], $W$ has the homotopy type of $Y \vee S$, where $Y$ is simply connected and $S$ is a finite wedge of circles. Given a fixed comultiplication $\psi$ on $Y$, then for every comultiplication $\mu$ on $S$ we obtain a comultiplication $(\psi, \mu)$ on $Y \vee S$ in the obvious way. Clearly, if $\mu$ is equivalent to $\mu^{\prime}$, then $(\psi, \mu)$ is equivalent to $\left(\psi, \mu^{\prime}\right)$. Conversely, it can be shown that if $(\psi, \mu)$ is equivalent to $\left(\psi, \mu^{\prime}\right)$, then $\mu$ is equivalent to $\mu^{\prime}$. The result follows from Lemma 7.5

\section{REFERENCES}

1. M. Arkowitz, Co-H-Spaces, in Handbook of Algebraic Topology (I.M.James, ed.), Elsevier Science, 1995, pp. 1143-1173. MR 96m:55012

2. M. Arkowitz and G. Lupton, Equivalence classes of homotopy-associative comultiplications of finite complexes, J. Pure Appl. Math. 102 (1995), 109-136. MR 96j:55008

3. J. Birman, An inverse function theorem for groups, Proc. Amer. Math. Soc. 41 (1974), 634638. MR 48:8632

4. I. Berstein and E. Dror, On the homotopy type of non-simply-connected co-H-spaces, Illinois J. Math. 20 (1976), 528-534. MR 53:11607

5. B. Eckmann and P. Hilton, Structure maps in group theory, Fund. Math. 50 (1961), 207-221. MR 24:A2612

6. _ Group-like structures in general categories III, Math. Ann. 150 (1961), 165-187. MR $\mathbf{2 7}: 3682$

7. R. Fox, Free differential calculus I, Ann. of Math. 57 (1953), 547-560. MR 14:843d

8. T. Ganea, Cogroups and suspensions, Inv. Math. 9 (1970), 185-197. MR 112:2484

9. P. Hilton, G. Mislin and J. Roitberg, On co-H-spaces, Comment. Math. Helv. 53 (1978), 1-14. MR 80d:55014

10. D. Kan, Monoids and their dual, Bol. Soc. Mat. Mex. 3 (1958), 52-61. MR 22:1800

11. W. Magnus, A. Karrass and D. Solitar, Combinatorial Group Theory, Wiley, 1966. MR 34:7617

12. J.D. Stasheff, H-space problems, H-Spaces, Springer-Verlag LNM 196, 1971, pp. 122-136. MR 44:4748

Department of Mathematics, Dartmouth College, Hanover, New Hampshire 03755

E-mail address: martin.arkowitz@dartmouth.edu

Department of Mathematics, Tufts University, Medford, Massachusetts 02155

E-mail address: mgutierr@tufts.edu 